\title{
THE DIFFERENT ADAPTIVE ALGORITHMS FOR ACTIVE VIBRATION CONTROL OF AUTOMOTIVE COMPONENT
}

\author{
Akash Gokul Bharate \\ Department of Mechanical Design Engineering, \\ RIT, Islampur, Maharashtra, India
}

\begin{abstract}
Modern control applications are becoming increasingly important in the area of vehicle ride and comfort. An attractive application in this area is to use of active vibration control (AVC). Many of the unwanted vibrations provides discomfort to operator of automobile. The vibrations also affect to the human and structure. The vibration contains wide range of frequencies which are difficult to control. There are different ways to control these active vibrations such as shunt damping and adaptive algorithms. In present Study, the different adaptive algorithms such as Filtered $x$ Least Mean Square (Fx-LMS), Normalized Least Mean Square (NLMS), Feedback Least Mean Square, two weighted notch FxLMS was studied for vibration control and system identification. The comparative study of these algorithms gives the different-different advantages and disadvantages. Among these algorithms the NLMS algorithm was very useful for system identification and two weighted notch Fx-LMS was useful for Control Logic.
\end{abstract}

Keywords - Active Vibration control, Fx-LMS, NLMS, Adaptive Algorithm, Feedback LMS, Signal processing.

\section{INTRODUCTION}

Most of the automotive vehicles and mechanical machines makes the noise and vibration. These noise and vibration are undesirable for machine health as well as for human health. Vibrations in automotive part arises due to external and internal forces. And these vibrations transmit to the structure of the automotive and then transmits to the body of the operator and passenger in vehicle. The continuous interaction of the body with vibration causes the several diseases like numbness, white finger syndrome (mainly seen in operator's hand), joint pain, muscular and neurological disorders. [1]. Similarly, these vibrations were harmful for the structure of the vehicle. The continuous vibration reduces the life of the component and also provide high risk failure of component in resonance condition. Many times, the loosening of nut and bolt, small cracks in welded part occurs due to the high amplitude vibrations.

For safety of the vehicle components and the human body parts, it was necessary to control the vibration in real time.

\author{
Dr. Ramchandra G. Desavale \\ Department of Mechanical Design Engineering, \\ RIT, Islampur, Maharashtra, India
}

There are different techniques for vibration control under categories like reduction of source of excitation, break the transmission path between source and receiver and control resonances and damp the vibrations at various frequencies using controlling strategies. In general, the vibration was damped using the force reduction, mass reduction, tuning, isolation and damping methods. In passive vibration control, the vibrations were damped with fixed and permanent material such as rubbers or mechanical springs, external masses etc. In active vibration control, the actuators and sensors were used to capture the vibration and produce the anti-vibrations to cancel out the incoming vibrations.

The active vibration control using smart material as actuator and sensor was beneficial when controlled by adaptive filters and algorithms. The vibration control using adaptive algorithm technique consist of two part as the system identification and control logic. The system identification means the behaviour replication of simple or complicated structure/ component into the array of the weights. Hence, whenever the same input was given to both (the real system/ structure and array of weights), it gives the similar output values of that behaviour. For this system identification, the different algorithms such as FxLMS, NLMS etc. was used. [4,5] The control logic was developed using two weighted notch filter, self-extraction LMS and feedback LMS.

\section{HARDWARE SETUP}

The fundamental block diagram of setup was shown in fig 1 . For active vibration control the frequency of the incoming vibration was important. For capturing the frequency sensors was used in present study. Also, the accelerometer sensor was used for measuring the incoming vibration. In present study, the vibration was controlled by producing the anti-vibration with piezoelectric actuator. But the important fact was how to decide the amplitude of anti-vibrations. For vibration control calculation the Adaptive algorithms such as FxLMS, NLMS, Feedback LMS, two weighted notch LMS/NLMS was used. $[2,3]$ 


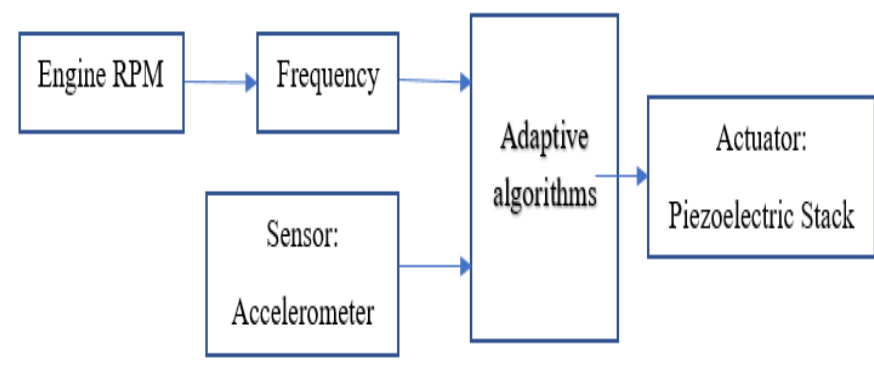

Fig. 1. Block Diagram of vibration control strategy.

\section{ADAPTIVE ALGORITHMS}

$O$ In active vibration control, the accurate production of anti-vibration was important. For this the adaptive algorithms were useful because of its self-adaption of weights using the gradient vectors. In present study the adaptive algorithms will be studied under System Identification and Control logic.

\section{A. System Identification -}

The system identification gives the replication of the complex system into the set of vectors. This was useful in multi ways such as it minimizes the complexity of structure, material properties and provide the easy way to process in software's. The structure of the System Identification process shown in figure 2. Depending upon the error value and input array of signal the weights was developed by algorithms and system was formed similar to primary system.

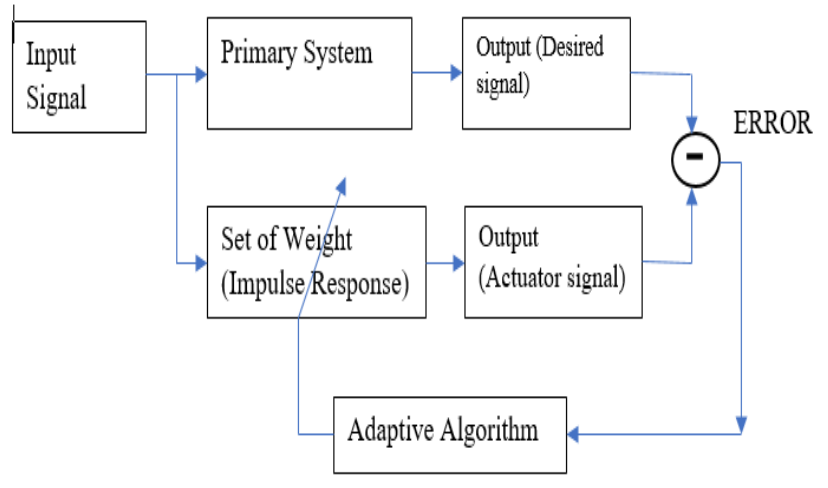

Fig. 2. Block Diagram of System Identification.

For this, the FxLMS and NLMS algorithms was used. Both of this algorithms work on the steepest descent gradient. The major difference of the above-mentioned algorithms was selfadjustment of the convergent factor. In FxLMS, the weights were adapted with the error produced by the difference of the actuator signal and actual desired output signal using equation 1 to equation 3 .

$$
\begin{aligned}
& \mathrm{e}_{\mathrm{k}}=\mathrm{d}_{\mathrm{k}}-\mathrm{y}_{\mathrm{k}} \\
& \left.\mathrm{MSE}=\mathrm{E}_{[} \mathrm{e}_{\mathrm{k}}^{2}\right]=\mathrm{E}\left[d_{\mathrm{k}}^{2}\right]-2 * \mathrm{P}^{\mathrm{T}} * \mathrm{~W}+\mathrm{W}^{\mathrm{T}} * \mathrm{R} * \mathrm{~W}
\end{aligned}
$$

where $\mathrm{E}[\cdot]$ represents a mean value, $\mathrm{P}=\mathrm{E}[\mathrm{dk} * \mathrm{ukT}]$ gives an input cross-correlation matrix, and $\mathrm{R}=\mathrm{E}\left[\mathrm{uk}^{*} \mathrm{ukT}\right]$ represents an input auto-correlation matrix. The mean square error function, shown within the Equation (2) could be a quadratic function of the filter weights. The adaptive filter changes its weights to search out the minimum MSE by finding a bottom of the bowl-shaped MSE surface where the MSE gradient is zero. The gradient vector of MSE is calculated by differentiating the MSE function with reference to filter weights. The strategy of steepest descent updates the weight vector consistent with Equation (3)

$$
\mathrm{W}_{\mathrm{k}+1}=\mathrm{W}_{\mathrm{k}}+\mu^{*}\left(-\nabla_{\mathrm{k}}\right)
$$

where $\mu$ represents a convergence factor/step size of the steepest descent algorithm. The steepest descent algorithm was developed using the estimated $\left(\nabla_{\mathrm{k}}\right)$ square errors of mean square error. From that the FxLMS algorithm was further developed.

In FxLMS, the convergence factor was used with a trial and error method. After every single value of $\mu$, the error between desired and actuator output signal was evaluated and depending upon the error the next $\mu$ value was decided and applied to check out the next error. It takes more time to identify the convergence factor for the particular analysis.

In NLMS, the weight adaption process was similar to the FxLMS algorithm but has major difference in convergence factor. In NLMS, the convergence factor was calculated by normalizing the input signal and with some constant to minimize the error of infinite output. The calculation of finding the convergence factor given by the equation (4).

$$
\mu=\sqrt[2]{\sum_{K=1}^{N}\left|x_{k}\right|^{2}}
$$

\section{B. Vibration Controlling Algorithms -}

In vibration control, the anti-vibration forming by an algorithm and actuator should be in real time. It means that every operating time of software, the accurate output values comes out by algorithm. In system identification the desired signal was measured with the help of accelerometer so that the weights can be adapted using desired and input signal offline or online way. But in control logic, the desired output was unknown. The accelerometer in setup will be gives the error value only. So, it was necessary to create a desired value or a reference value using the orthogonal properties of signal and current frequency of input signal. After that, adapt the weights using the measured error and reference signal and damp the 
vibration. In this logic, the input signal and its properties like frequency etc are the only input. The outline of control logic given in figure (3).

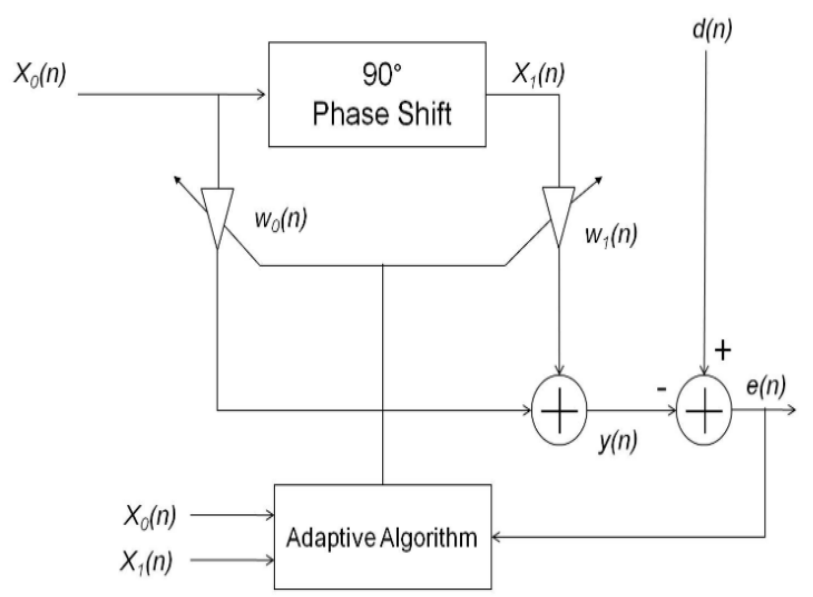

Fig. 3. Block Diagram of Control Logic.

In control logic, there were two different algorithms used. The two-weight notch LMS algorithm and normalized LMS.

The two-weight notch LMS gives the output value for each processing time using the sin and cos function for its reference signal generation. The weight adaption was done by the similar calculation of LMS algorithm but the size of the weights was different. Only two weights were used to calculate the output and this were adapted by error difference and input signal.

In the NLMS, the weight updating and the calculation of output signal was similar to the LMS but convergence factor was calculated by normalizing the input data array. In this, the weight size should be greater than two or some greater sized array was used so that the normalizing will be done respect to number of wights.

In present study, all algorithms were made in MATLAB software and analysis with different type of signals such as harmonic vibration ( $\sin$ wave), random signal vibration, square wave. Comparative study was done in system identification and control logic for better performance.

\section{RESULTS AND DISCUSSION}

\section{A. System Identification}

In present study, the system identification simulation was done in MATLAB with taking the two different signals like sine wave for simply understanding and random signal/vibration was taken for checking the versatility of algorithm for any type of signal. The FxLMS and NLMS algorithm was used for development of weights of signal.

In figure (4-a), it was seen that the sine wave given as the input signal and another sine wave with different amplitude was taken as desired signal. After the calculation by FxLMS algorithm the outcome coming from it was also shown in figure (4-a). The error of actual and algorithm output also seen in it. The values of error shows that the error was nearly zero mean exact antivibration was produced. Also, in figure (4-b), when the random signal was given as input then the error was seen in the graph. The error values were lying about zero but it hasn't exacted zero value. It shows that the FxLMS was not exactly matches with desired signal still it gives the good result for identification in random signal.
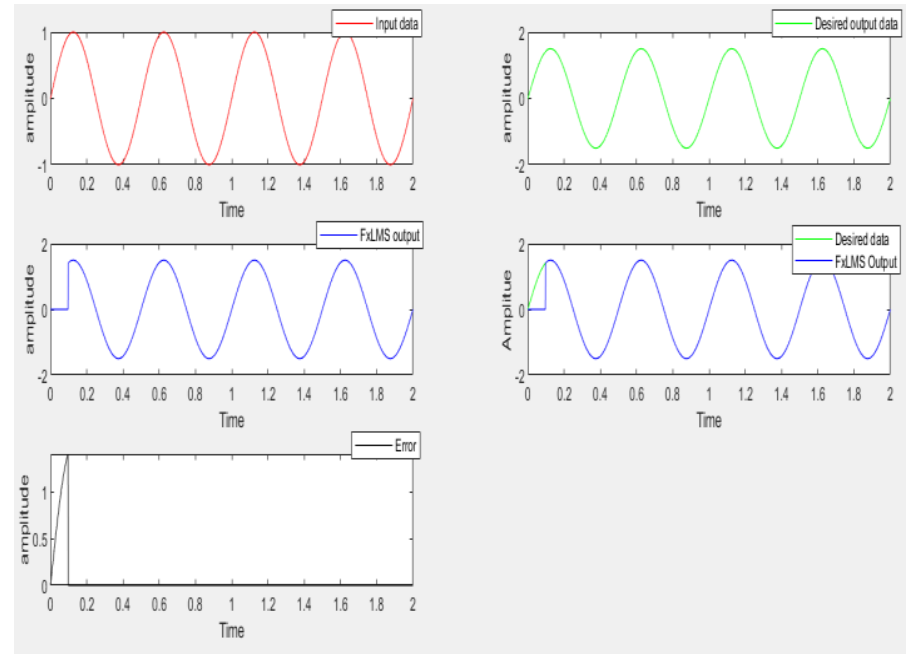

(a)
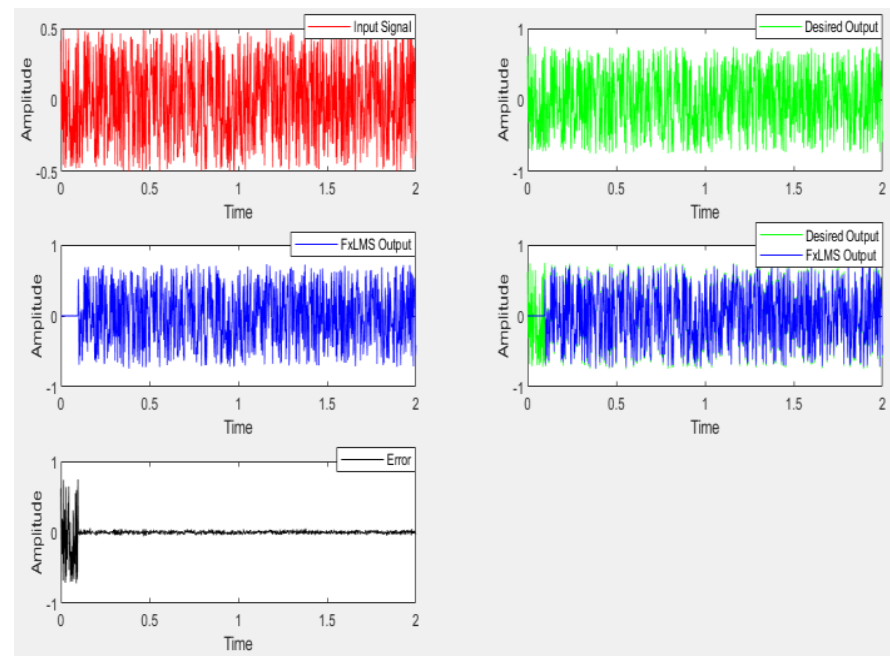

(b)

Fig. 4. System Identification with Fx-LMS (a) with sin wave (b) with random signal.

In figure (5-a), the similar signal was given to NLMS algorithm with same desired output. It was seen that the algorithm output was developed in very quick time so that the error graph in figure (5-a) shows that the error was exactly zero after one or two steps of updating of weights. Also, the 
error value was seen exactly zero after few iterations of calculation when the random signal was given to the NLMS algorithm.

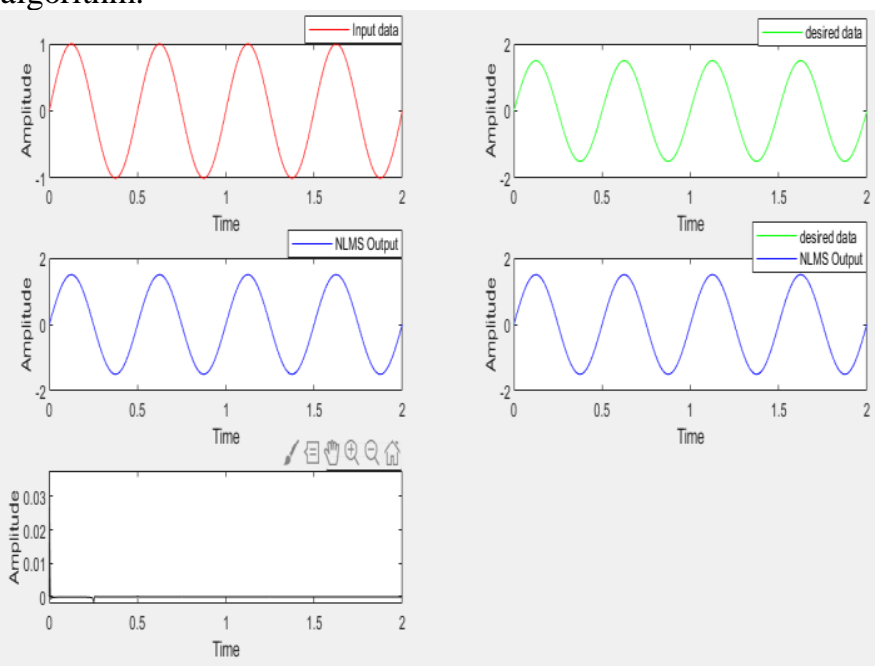

(a)
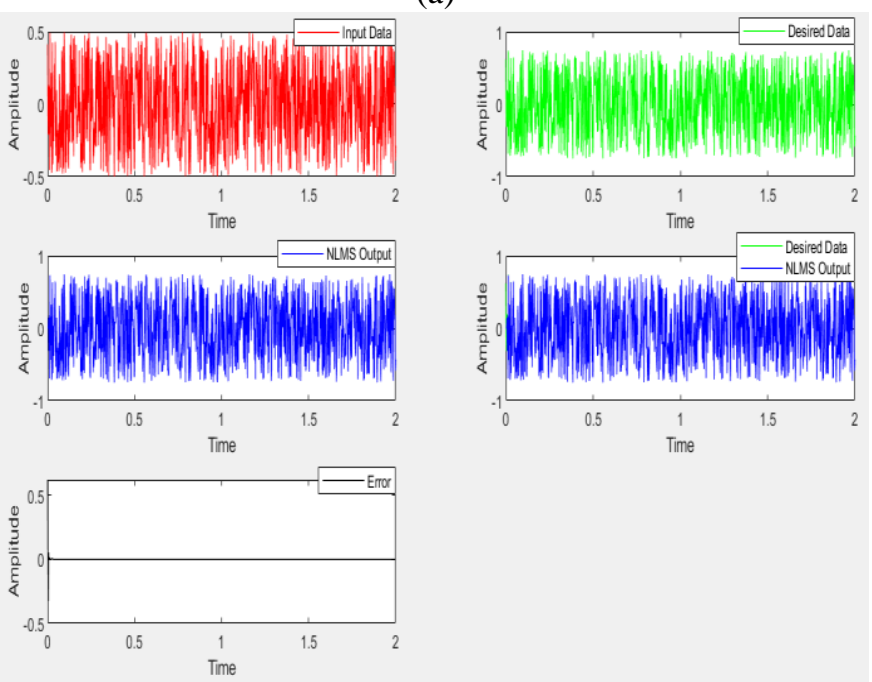

(b)

Fig. 5. The System Identification with NLMS algorithm (a) using Sine wave (b) using random signal.

\section{B. Control Logic}

In vibration control the time was very less for producing the anti-vibration because continuous vibrations were coming from source with different amplitude. For smooth operation and less processing time, it was needed to store less data. Most difficult task was to calculate the upcoming vibrations and in this it was solved by using this algorithm.

In figure (6-a), it was observed that the sine was given as the incoming signal to the notch LMS algorithm. The desired signal was given after the incoming signal was reached after the system developed in system identification (set of weights). The error value shows that the accuracy of the algorithm. It gives the zero error after quick time of processing. In figure (6b), it was observed that the error was too much small nearly about zero when the random signal given to the Notch LMS algorithm. The Notch LMS produces the 95\% antivibration(signal) matching with desired signals.
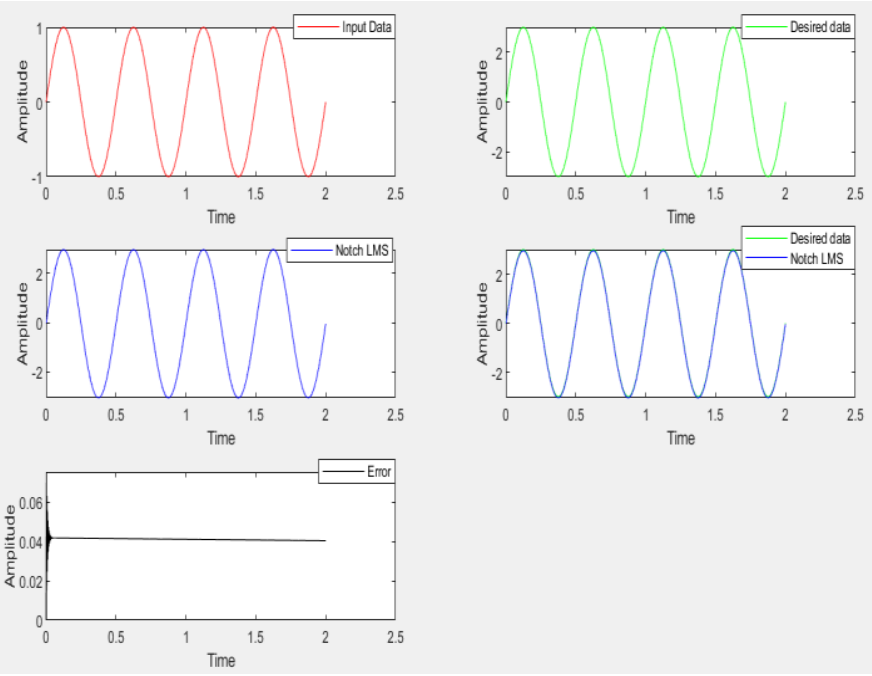

(a)
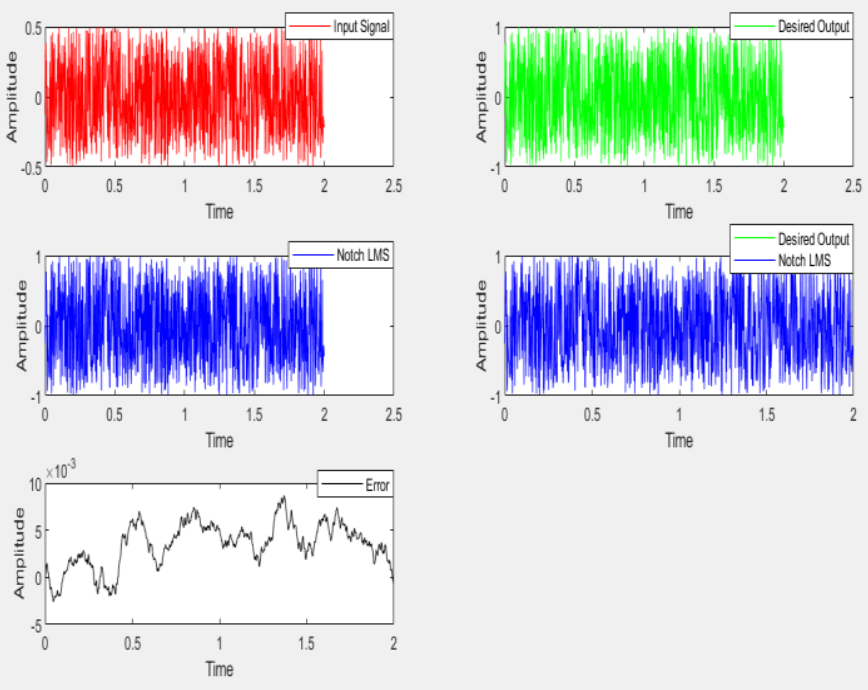

(b)

Fig. 6. The Control Logic with Notch-LMS algorithm (a) using Sine wave (b) using random signal.

In figure (7-a), the sin wave was given to the NLMS algorithm and was observed that the error value becomes the zero after some iteration. But the NLMS needs the large data store for calculation which makes the time taking process for large processing data. In figure(7-b), it was observed that the error was not becoming the zero for whole time but it produces the close matching anti-vibrations. The NLMS algorithm gives 
the $75 \%$ anti-vibration was matching with the desired signal for damping.
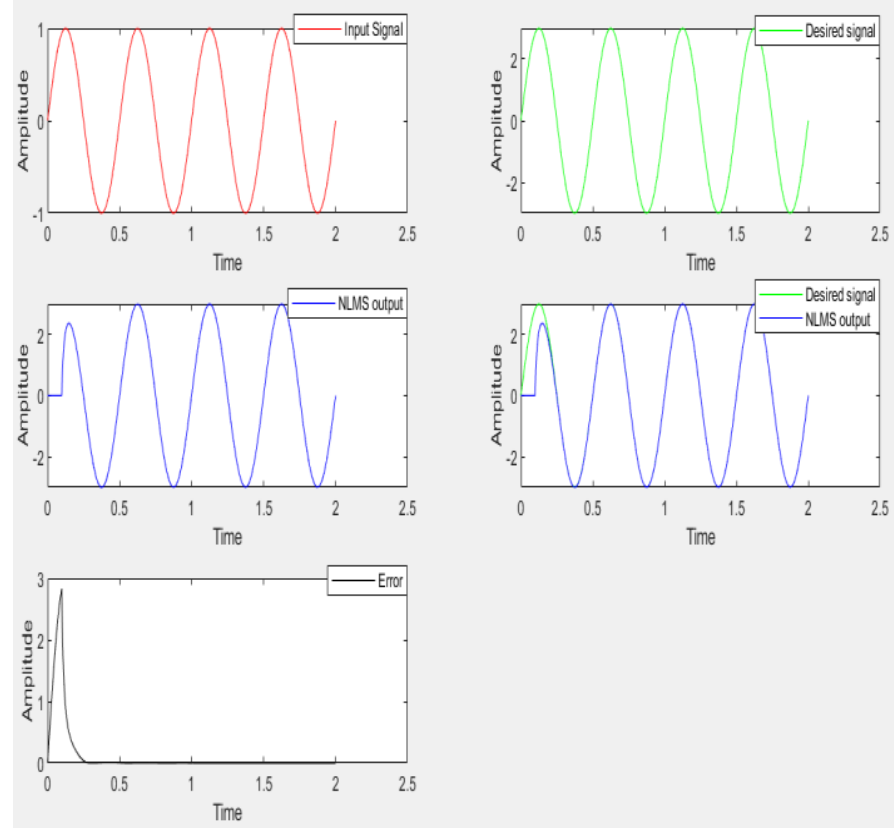

(a)
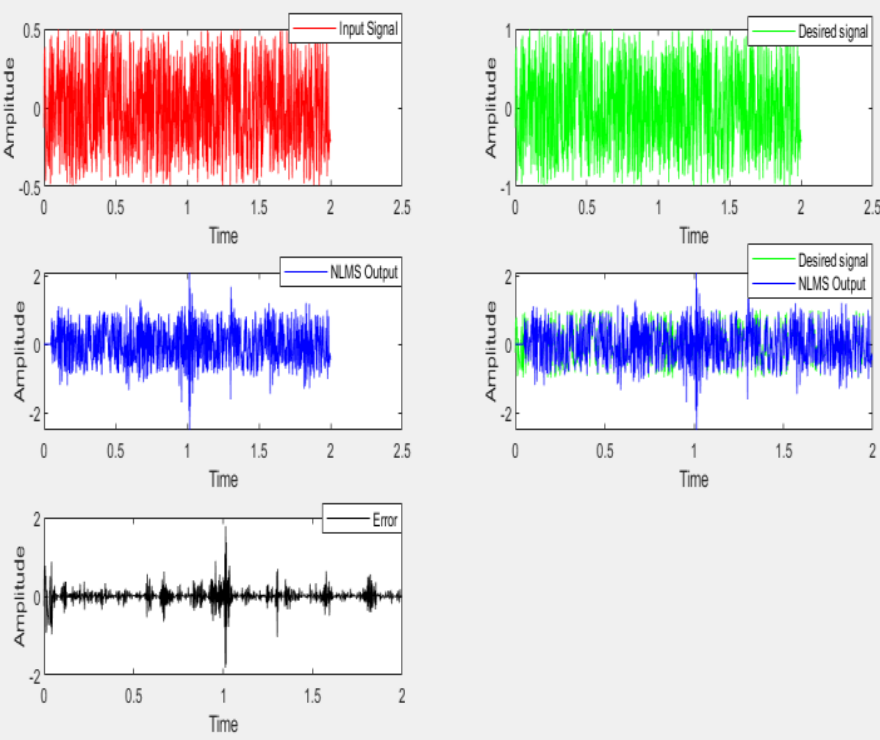

(b)

Fig. 7. The Control Logic with NLMS algorithm (a) using Sine wave (b) using random signal.

\section{CONCLUSION}

In present study, it was observed that the both the algorithms were effectively work for the system identification and the control logic. From the observation it was concluded that the NLMS algorithm was more useful for the system identification because convergence factor was automatically calculated and gives the better result in less iteration. But FxLMS also provide good results but convergence factor should be calculated with trial and error method.

From the results and the observation from graphs, it can be concluded that the Notch LMS was useful for the control logic of vibration because it has capability of producing exact antivibration to damp the incoming vibrations with two weights. The NLMS algorithm was not much suitable for the controlling the vibrations.

From above it was concluded that the NLMS algorithm for system identification and Two weight notch LMS algorithm for control logic was the best choice in active vibration controlling in automotive.

\section{REFERENCE}

[1] Dong R.G., Schopper A.W., McDowell T.W. et.al. (2004). "Vibration energy absorption (VEA) in human fingers-hand-arm system", Medical Engineering \& Physics, Elsevier, PP: 483-492.

[2] Saurabh R. P.,and Godbole B. B..(2018).“Optimization of LMS Algorithm for System Identification”, Research Gate, PP: 1-14.

[3] Bipin K., and Manal U.. (2015). "Control of Vibrations using Adaptive Filters", International Science Press, PP: 1243-1249.

[4] Wenchao Niu, Chengzhe Zou and et.al. (2019). "Adaptive vibration suppression of time-varying structures with enhanced FxLMS algorithm", Mechanical System and Signal Processing (ELSEVIER), PP: 93-107.

[5] Arunkumar P., Bharanidharan K. et.al.. (2014). "A Design Approach of Normalized Least Mean square (NLMS) Algorithm for Low Power Implementation of Adaptive Noise Cancellation”, IJRASET, PP: 172-177.

[6] Walter j Kozacky and Tokunbo Ogunfunmi. (2013). “An active noise control algorithm with gain and power constraints on the adaptive filter", EURASIP Journal on Advances In signal Processing, PP: 1-12.

[7] Gao Shouwei, Huang Quanzhen et.al.. (2010). “A Feedback LMS Adaptive for Active Vibration Control",World Congress on Intelligent Control and Automation, PP: 4885-4890.

[8] Sang-Kwon Lee, Seungmin Lee et.al..(2018).“A new Method for Active Cancellation of Engine Order Noise in a Passenger car", Applied Sciences, PP: 1-22.

[9] Quanzhen Huang, Jun Luo et.al.. (2014).“Analysis and Implementation of adaptive filtered-XLMS algorithm based on reference signal self-extraction", JVE International Ltd., PP: 2341- 2354. 
[10] Marko Hoyhtya and Aarne Mammela. (2016). "A unified framework for adaptive inverse power control", Springer EURASIP Journal, PP: 1-15.

[11] Dariusz bismor and Marek Pawelczyk. (2016). "Stability Conditions for the Leaky LMS Algorithm Based on Control theory Analysis", PAN Archives of Acoustics, PP: 731-739.

[12] Radwin R. G., Thomas J. A., and Don B. C.. (1990). "Hand-Arm Frequency Weighted Vibration Effects On Tactility", International Journal of Industrial Ergonomics, Elsevier, PP: 75-82.

[13] Muhammad W., and Gupta N.. (2014). "Design and Implementation of Least Mean Square Adaptive Filter on Fetal Electrocardiography", ASEE, PP: 14-19.

[14] Mitra Anirban., Akash Bharate et.al.. (2018). "Experimental Vibration Analysis of Isolator Material on Hand-Handle Interface for CHOPSAW Machine." Materials Today: Proceedings 5, no. 2.

[15] Mitra, Anirban, Bharate, Akash et.al. (2018). "Experimental Vibration Analysis of Isolator Material on Hand-Handle Interface for CHOPSAW Machine", Materials Today: Proceedings, 5(2), 54385444. 